\title{
Alignment of a three-mirror telescope using the sine condition test
}

\section{David Sommitz, Matthew Dubin}

David Sommitz, Matthew Dubin, "Alignment of a three-mirror telescope using the sine condition test," Proc. SPIE 10747, Optical System Alignment, Tolerancing, and Verification XII, 1074702 (19 September 2018); doi: 10.1117/12.2320689

EDIE Event: SPIE Optical Engineering + Applications, 2018, San Diego, California, United States 


\title{
Alignment of a three-mirror telescope using the sine condition test
}

\author{
David Sommitz*, Matthew Dubin \\ College of Optical Sciences, University of Arizona, Tucson, Arizona, 85721, USA
}

\begin{abstract}
The Sine Condition Test has been experimentally demonstrated as an effective tool for measuring linearly field dependent aberrations in simple optical systems. Simulations have also shown that it can be used to provide feedback as part of an alignment procedure for more complex systems. In this paper we show how the Sine Condition Test was used as part of the process for aligning a three mirror telescope. We present the basic concept of the Sine Condition Test, how it was implemented in our system and the experimental results from multiple alignments. Finally, we compare our experimental results to simulated results.
\end{abstract}

Keywords: Sine Condition Test, Abbe Sine Condition, TMA, Alignment

\section{INTRODUCTION}

The sine condition is conventionally used to describe the field-dependent aberration content of an optical system. Systems are talked about in terms of whether their design satisfies the sine condition. Recent work has focused on the concept of using the sine condition as a tool for measuring the state of alignment of an optical system ${ }^{1}$. The sine condition test (SCTest) has been developed as an alignment technique for optical systems for which a single on-axis measurement is insufficient for alignment ${ }^{2}$.

This work functions as an experimental validation of the SCTest for a multi-element optical systems, previously theorized and simulated by Dr. Lampen at the University of Arizona ${ }^{3}$. The system needed to have significant complexity without becoming impractical, so a three-mirror telescope with no rotational symmetry was chosen as the unit under test (UUT). It needed to be a system with wavefront performance that changed significantly with misalignment without being impossible to align with current techniques. Finally, it needed to be a system that had significant enough fielddependent aberrations from misalignment so as to require an additional measurement to improve alignment. The experiment was performed by aligning the UUT as well as possible using conventional means. The detailed procedure is also discussed along with in-depth analysis of results and predictability by Sommitz ${ }^{4}$. The UUT was then misaligned by perturbing the mirrors by a randomly generated but known amount. Relative changes in wavefront error were measured and simulated. This measurement was used to calculate an adjustment to the mirrors to improve the alignment.

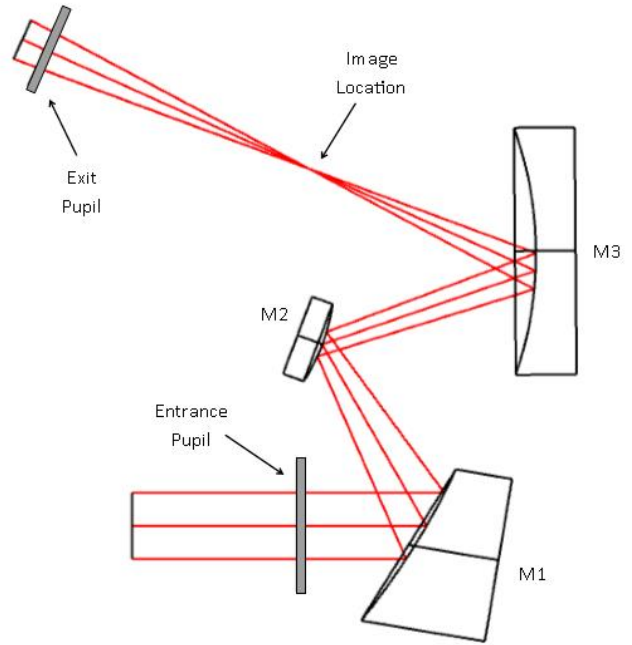

Figure 1. Two-dimensional layout of nominal design. 


\section{BACKGROUND}

\subsection{Abbe sine condition}

The Abbe sine condition describes the ideal mapping between ray angles in object and image space. The relation between the two angles is given by

$$
\sin \left(\theta_{o b j}\right)=m \sin \left(\theta_{i m g}\right)
$$

where $\theta_{\text {img }}$ is the angle of the ray in image space referenced to an arbitrary direction, $\theta_{\text {obj }}$ is the angle of the same ray in object space, and $\mathrm{m}$ is the constant magnification term between the two. When this mapping is satisfied in an optical system, it can be shown through Fermat's principle that the system will have no linearly field-dependent aberrations ${ }^{2}$. Measuring the pupil mapping error is useful because it gives information about the system misalignment due to the presence of linearly field-dependent astigmatism in misaligned systems ${ }^{1}$.

\subsection{Sine condition test}

In its general form, the SCTest measures whether a system violates the Sine Condition. This is any sort of test that measures the pupil mapping error of an optical system (see Lampen ${ }^{2}$ for the relation between pupil mapping error and the Sine Condition). This could be done in a variety of ways- one would be to place a grid of dots at the entrance pupil and take an image of the exit pupil. The SCTest could also be administered with a single point source that can be moved and reliably tracked around the pupil. These relatively simplistic methods are prohibitive for this research project due to their time-consuming nature and limited resolution.

\section{METHOD: PROCEDURE SUMMARY}

\subsection{Sensitivity matrix}

The linear sensitivities of the wavefront can be conveniently represented by a matrix where the rows represent the sensitivities of a specific Zernike polynomial coefficient to each of the degrees of freedom and the columns represent the sensitivities of each Zernike polynomial to a specific degree of freedom. This sensitivity matrix can be determined by making small perturbations to each of the degrees of freedom and tracking the change in Zernike polynomials. The individual sensitivities in this experiment were found to be linear to within $1 \%$ at a perturbation size of $1 \mathrm{~mm}$ or $1^{\circ}$. In other words, the resultant Zernike terms from a component displacement of $1 \mathrm{~mm}$ could be predicted by the linear sensitivities with an error of less than $1 \%$. The high degree of local linearity in the sensitivities means that the sensitivity matrix can be pseudo-inverted to prescribe adjustments to improve the alignment of coarsely aligned systems.

\subsection{Singular value decomposition}

A pseudo-inverse of the sensitivity matrix is necessary when the matrix is non-invertible. Obtaining a pseudo-inverse of a matrix can be thought of as making a least squares approximation of the matrix. A common solution to a least squares problem is singular value decomposition (SVD), which rearranges the matrix into an invertible form ${ }^{5}$. SVD is a linear algebraic manipulation that decomposes and rearranges a single matrix, $\mathrm{J}$ into three matrices, $\mathrm{U}, \mathrm{W}$, and $\mathrm{V}$. The three matrices can be recombined back to form the original matrix in the following way, where $\mathrm{V}^{\mathrm{T}}$ is the transpose of $\mathrm{V}$ :

$$
J=U W V^{T}
$$

$\mathrm{W}$ is arranged with descending singular values of $\mathrm{J}$. The singular vectors of a matrix make up the basis of the matrix. In other words, they are a set of linearly independent (orthogonal) vectors that combine to make up the entire matrix. Each singular vector has an associated weight or singular value. Singular vectors with large singular values are strongly present in the matrix.

\subsection{Compensator selection}

When the measurement noise of an optical system and its nonlinearity are well known, the information that SVD provides enables the designer to set a threshold below which the degrees of freedom are ignored during realignment. It is not sufficient, however, to look at the matrix $\mathrm{W}$ for this information. That is because it is a combination of the most sensitive degrees of freedom and the most 'sensitive' (highly prominent) Zernike terms. The procedure outlined in Chapman and Sweeney ${ }^{6}$ describes how to perform a second SVD on the W and V matrices (ignoring the U matrix since 
it contains information about the individual Zernike weights) to reduce the sensitivity matrix, starting by eliminating the least sensitive DOF and stopping when the threshold is reached.

\subsection{Sine condition test measurement}

The UUT is measured by placing a point source in finite conjugate (image) space, so this particular system is measured backwards. See section 3.5 for further discussion. The Ronchi ruling at the exit pupil functions as the projection grating. An order selection aperture is placed at the system focus to block the unwanted orders. A second Ronchi ruling is placed at the aperture stop and functions as the analyzer grating. The remaining components form the imaging subsystem. An objective lens is placed at the aperture stop, a fold mirror directs the beam toward a $50 \mathrm{~mm}$ field lens mounted in a cage system with the camera (CCD). An additional order selection aperture is placed before the field lens to block the remaining unwanted diffracted beams.

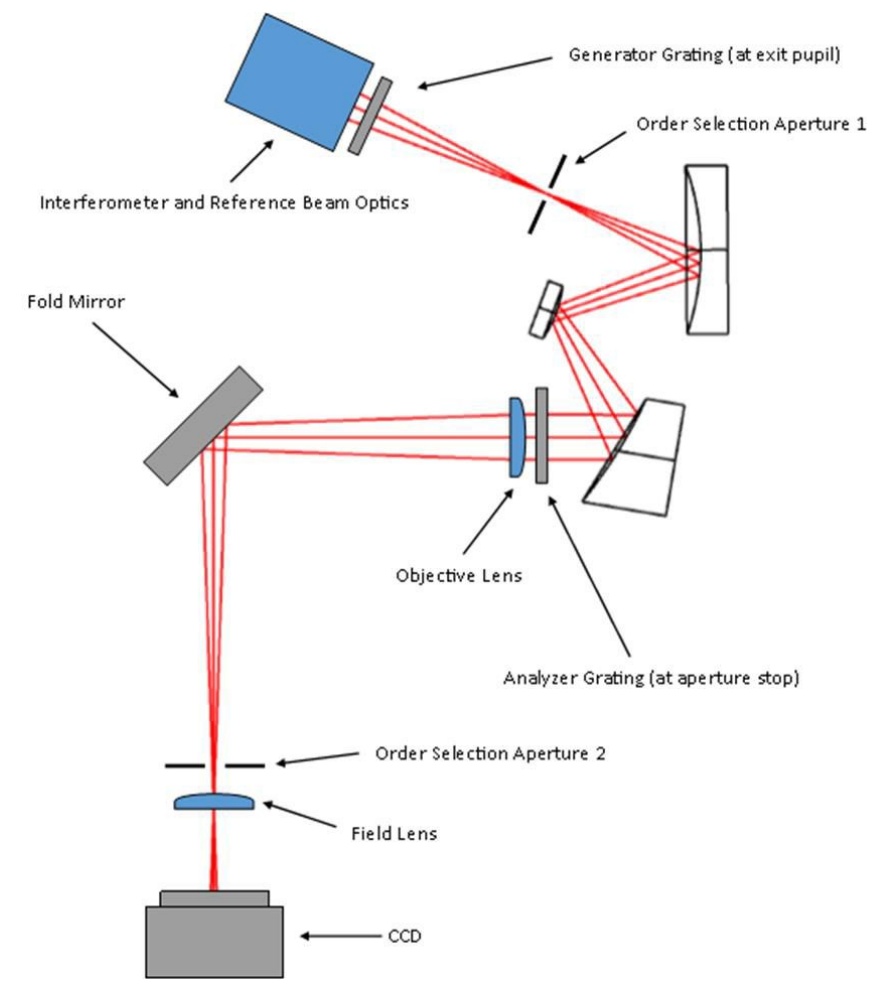

Figure 2. Layout of the SCTest

\subsection{On-axis test}

The on-axis test is a double-pass measurement performed from the image space to object space of the telescope. A flat mirror is placed in the path of the collimated beam in object space in order to redirect the beam back through the system. An alignment of the optical system can be performed by measuring the system in either direction: a collimated beam can be directed toward the entrance pupil and a reference sphere placed at the system focus. Alternatively, a diverging beam can be directed toward the exit pupil and a reference plane mirror can be placed in collimated space. If the system only needs to be aligned once, then changing direction will not impact the complexity of the procedure. The reverse direction was chosen for this project since the system was aligned multiple times: finely adjusting two degrees of freedom to redirect the beam in collimated space is easier than controlling three in the finite conjugate space.

\subsection{Initial system alignment}

The experiment required a well-aligned initial alignment state. To initially position the components, an interferometer was used in tandem with a coordinate measurement machine with a point source microscope attachment. Nominal alignment was chosen based on arbitrary sensitivities to keep the maximum wavefront error over the field less than $300 \mathrm{~nm}$. 
The accuracy of the CMM was measured by testing absolute error, single measurement repeatability, and squareness. The coordinate measurement machine's absolute calibration was verified by measuring the lengths of well-defined gage blocks. Less than $6 \mu \mathrm{m}$ of absolute error were measured for various lengths of gage blocks up to $100 \mathrm{~mm}$. Single measurements were made repeatedly with a standard deviation of $1.3 \mu \mathrm{m}$. Lastly, the squareness of the CMM was observed by rotating a bar with a ball on either end and measuring the length several times. These measurements confirmed the calibration of the CMM and ensured a successful initial alignment.

\section{CONSIDERATIONS FOR SCTEST}

\subsection{Experimental design considerations}

Several features were considered in the design process. In general, since the SCTest has been implemented with singleelement systems, a system with higher complexity is desirable. Because simpler systems are easier to align, optics with fewer planes or axes of symmetry were preferred in order to demonstrate a more difficult alignment challenge. Consequently, systems using multiple spherical mirrors were avoided. A system with external (physically accessible) pupils was desirable since the gratings had to be placed at the pupils. Because an order-selection aperture must be used to block unwanted beams in the system, an intermediate focus was also desirable. A three-mirror telescope with a rectangular field, representative of common designs used in spectrometry applications, was used in this experiment. These parameters are not required for a successful implementation of the SCTest but were merely chosen to simplify the procedure.

\subsection{Measurement uncertainty}

The system can never be perfectly measured. The degree of uncertainty in the measurements for this alignment can have a significant impact on the improvement of the system misalignment. Sources of measurement uncertainty may vary for the individual alignment task. Three sources of measurement uncertainty are included in this paper: alignment error, region-of-interest (ROI) placement, and residual distortion.

Alignment uncertainty is based on the repeatability of the micrometers and tip/tilt stages. These were measured and verified with a CMM. The RMS wavefront error contribution for the alignment error was measured to have a standard deviation of $25.5 \mathrm{~nm}$. This error could be improved with optomechanical instruments with finer precision and higher accuracy.

ROI placement error describes the measurement error from the positioning of the ROI in software. The standard deviation of the RMS wavefront error contribution was $11.4 \mathrm{~nm}$. This uncertainty can be improved by adding a software routine that calculates ROI from phase shifted data rather than visually re-positioning the mask.

Finally, residual distortion is a measurement error that arises from distortion in the imaging subsystem. If the distortion in the imaging subsystem is known precisely, this feature can be corrected in post-processing. Alternatively, an imaging system with additional complexity can be used to further reduce the distortion. Measurement error from this effect is found to have a standard deviation of $6 \mathrm{~nm}$. See section 4.6 for additional discussion.

The sources of uncertainty are assumed to be uncorrelated and the RSS of all three errors has a $2 \sigma$ value of $57.1 \mathrm{~nm}$. This uncertainty can be used as the error bars in the experimental results.

\subsection{Ray aiming}

When generating the sensitivity matrix, ray aiming should likely be used in the lens design software. This significantly slows the simulation software and is especially problematic if large sets of Monte Carlo simulations are desirable during the design phase. If there are significant differences between the sensitivity matrices generated with and without ray aiming (see Section 3.1), the matrix obtained with ray aiming on should be used.

\subsection{Selection of Zernike terms for measurement}

There are a limited number of usable Zernike terms in the SCTest. Piston is ignored as well as X and Y tilt fringes. Information about the system's magnification error from tilt fringes can be used for alignment but the gratings and pupils must be precisely located to make these coefficients meaningful. Terms above astigmatism were not used since the amount of change expected was smaller than the measurement uncertainty for each of these terms. In other words, vertical and horizontal coma were ignored for the SCTest because they were below the expected noise floor. If components below the noise floor are used, the SCTest output adjustments will often be unrealistically small. 


\subsection{Order selection}

The unwanted diffracted orders from the projection grating must be removable even in an aligned state. If the nominal diffraction orders are only separated by a small amount, it is likely that the misaligned state will have spot patterns that overlap. During the design phase, it is important to simulate several aberrated spot patterns in order to ensure that the unwanted orders can be blocked in all cases.

\subsection{Imaging subsystem distortion}

The imaging subsystem has two primary functions- separate and block additional diffracted orders and image the fringe pattern at the pupil of the UUT onto the imaging system camera. While this can be done with a single lens, it is likely necessary to add at minimum a field lens to reduce the system distortion. Without this step, Zernike polynomials of one type will be coupled into other terms. Unless the amount of distortion is known to a high degree of certainty, correcting this effect with software is not recommended.

\section{RESULTS}

To appropriately characterize the entire procedure of the SCTest, the central field measurement results are combined with SCTest results in a single plot. The RMS wavefront error plotted is obtained by calculating the offset of the central field Zernike terms and the SCTest Zernike terms from their nominal values. The errors presented in this section's figures have the ideal wavefront subtracted out since there is non-zero wavefront error in the nominal design. The terms from the individual measurements are combined via root-sum-square and then both measurements are added together. The dashed lines represent the expected relative changes in Zemax and the solid lines represent the wavefront measured in the lab.

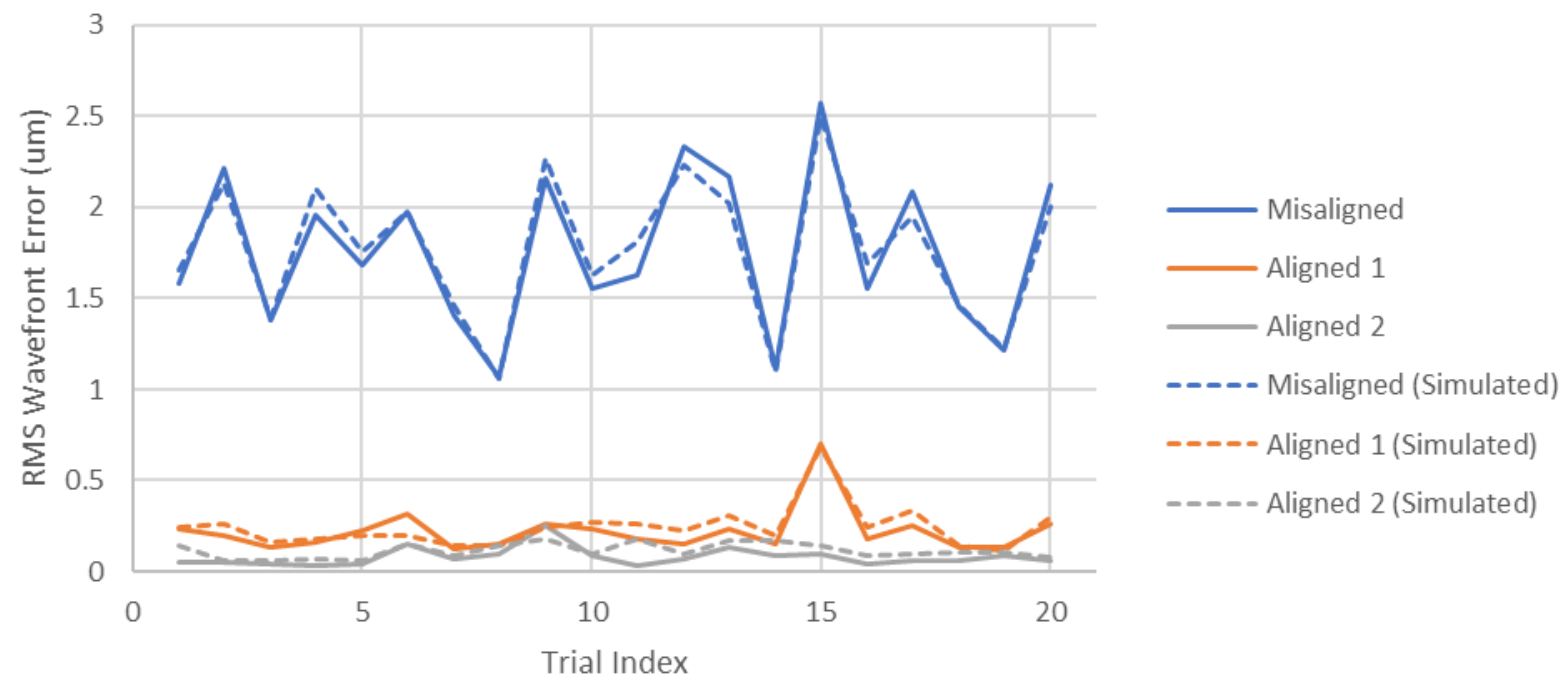

Figure 3. Residual wavefront error of combined on-axis and SCTest measurements, plotted by trial.

Detailed results are discussed in-depth by Sommitz ${ }^{4}$. Results of the SCTest are shown with expected values. 


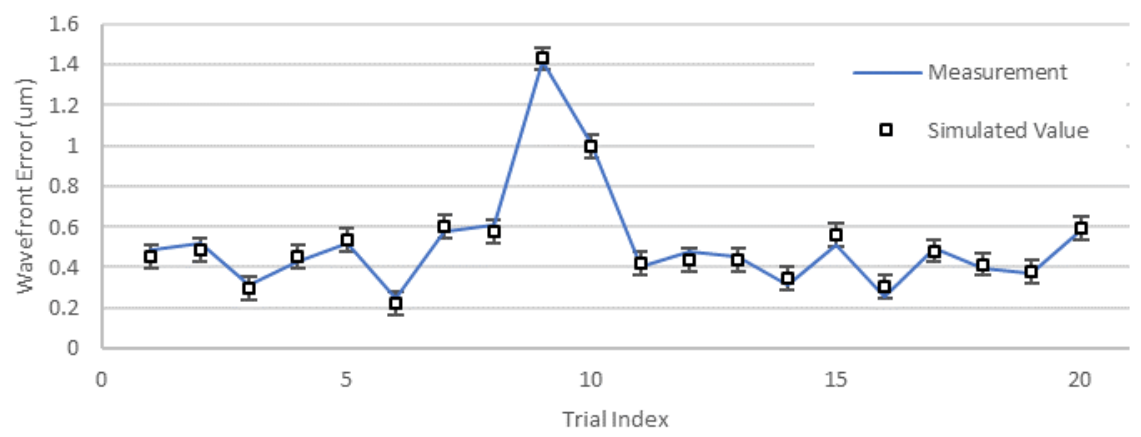

Figure 4. Residual RMS wavefront error for misaligned state of experiment. Error bars shown are the expected $2 \sigma$ range of error assuming an un-correlated normal distribution for each source of uncertainty. Figures and text similar to Sommitz ${ }^{4}$

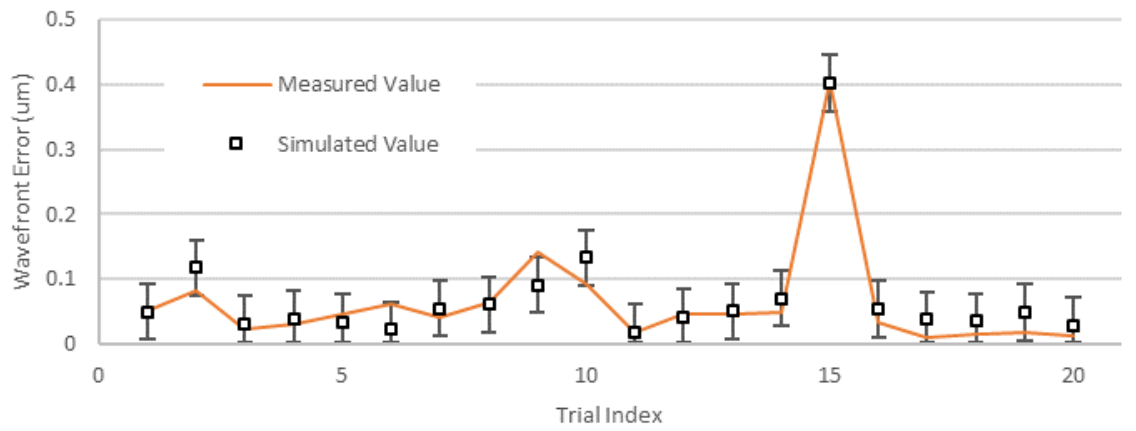

Figure 5. Residual RMS wavefront error after one iteration of alignment. Figures and text similar to Sommitz

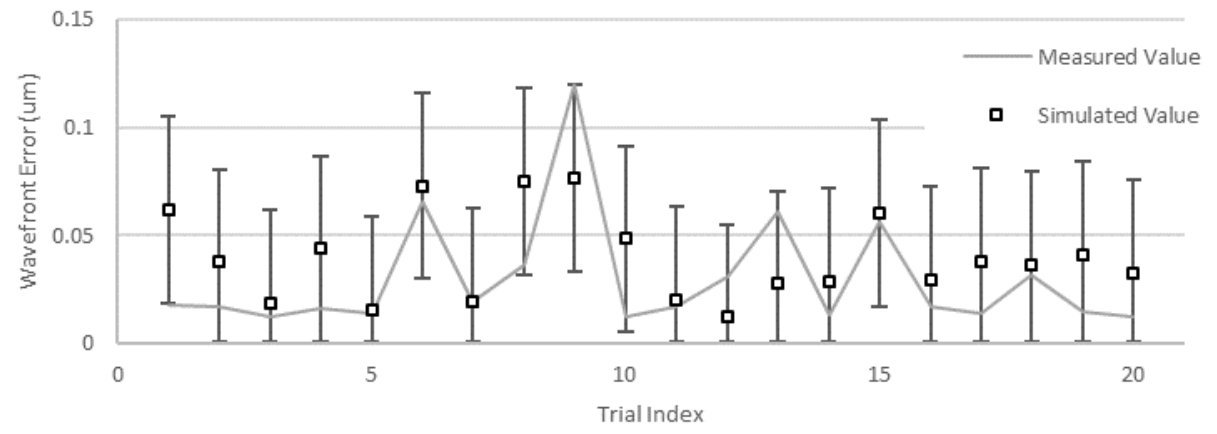

Figure 6. Residual RMS wavefront error after two iterations of alignment. Figures and text similar to Sommitz

The measured values match the expected values to within the uncertainty of measurement. A third iteration of the SCTest was not applied because results indicated there was no significant improvement after the second iteration. 


\section{CONCLUSION}

The theory of the SCTest previously laid out by Lampen ${ }^{3}$ showed via simulation that the SCTest is useful for significant improvement in alignment even when expected sources of noise in the system are included in the simulation. The results of the experiment confirm the theory and demonstrate the effectiveness of the SCTest in two ways. First, the wavefront error has been demonstrated to improve significantly over the field for each trial. Secondly, the changes brought about by each step of the experiment are shown to be predictable to within measurement uncertainty. This experiment demonstrates the feasibility and relative simplicity of the SCTest as a promising option for the alignment of complex systems.

\section{REFERENCES}

[1] J.H. Burge, C. Zhao, and S.H. Lu, "Use of the abbe sine condition to quantify alignment aberrations in optical imaging systems," Proc. SPIE, Vol 7652, p. 765219 (2010)

[2] S. Lampen, M. Dubin, and J.H. Burge, "Implementation of sine condition test to measure optical system misalignments,” Appl. Opt. 50, 6391-6398 (2011)

[3] S. Lampen, M. Dubin, and J.H. Burge, "Alignment of a three-mirror anastigmat using the sine condition test," Appl. Opt. 53, 1874-1888 (2014)

[4] D. Sommitz and M. Dubin, "Practical implementation of the sine condition test" OSA App. Opt. (to be published)

[5] C.L. Lawson and R.J. Hanson, [Solving least squares problems], Society for Industrial and Applied Mathematics, New Jersey, (1995)

[6] H.N. Chapman and D.W. Sweeney, "Rigorous method for compensation selection and alignment of microlithographic optical systems," Proc. SPIE, Vol. 3331, pp. 102-113 (1998) 